\title{
PULSATILE DRUG DELIVERY AS MODIFIED RELEASE DOSAGE FORM: A REVIEW
}

\author{
Gupta Nitan Bharti ", Sharma Pooja, Bhandari Neeraj, Singh Kulwinder, Kumari Asha
}

Department of Pharmaceutics, Sri Sai College Of Pharmacy, Badhani, Pathankot, Punjab, India - 145001

*Corresponding author E-mail: nitanbharti@yahoo.com

Received 25 Sep 2012; Review Completed 06 Oct 2012; Accepted 01 Nov 2012, Available online 15 Nov 2012

\begin{abstract}
Pulsatile drug delivery is a advanced drug delivery system in pharmaceutical field . Pulsatile system is one of the modified release dosage form system and gaining a lot of interest as it is increasing patient compliance by means of providing timeand site-specific drug delivery system. Pulsed or pulsatile drug release is defined as the rapid and transient release of a certain amount of drug molecules within a short time-period immediately after a predetermined off-release period. In various diseases in which we can recomend the pulsatile drug delivery system such as duodenal ulcer, cardiovascular diseases, arthritis, asthma, diabetes, neurological disorder, cancer, hypertension and hypercholesterolemia. This article summarize the various methodologies, evaluation parameters and compile the recent literatures with special focus on the different types and approaches involved in the development of the formulation

Keywords: Pulsatile drug delivery, Modified release dosage form, Chronopharmacotherapy, Site-specific drug delivery.
\end{abstract}

\section{INTRODUCTION}

Pulsatile system is amongst one of them and gaining a lot of interest as it is increasing patient compliance by means of providing time- and site-specific drugdelivery system, thus providing special Pulsed or pulsatile drug release is defined as the rapid and transient release of a certain amount of drug molecules within a short time-period immediately after a predetermined off-release period ${ }^{1}$. Pulsatile release is commonly found in the body, for example during hormone release, in which a baseline release is combined with pulsed, one-shot type release within a short time rangeand temporal delivery. A pharmaceutical dosage form such as a capsule capable of delivering therapeutic agents into the body in a timecontrolled or position-controlled pulsatile release fashion, is composed of a multitude of multicoated particulates (beads, pellets,granules, etc.) made of one or more populations of beads. On which cases or circumstance pulsatile drug delivery is used they are listed below.

1) Chronopharmacotherapy of diseases which shows circadian rhythms in their pathophysiology.

2) Avoiding the first pass metabolism e.g. protein and peptides $^{2}$

3) For which the tolerance is rapidly exists,

4) For targetting specific site in intestine e.g. colon,

5) For time programmed administration of hormone and drugs,

6) For drugs having the short half life and their mechanism.

Drugs which exhibit tolerance that drugs not be delivered at a constant rate, since the drug effect decreases with time at constant drug level. The drug toxicity increases with time. In such cases it is preferable for dosage form which will provide desired concentration of drug at particular time point only. Now the concept of chronopharmaceutics has emerged, where is research devoted to the design and evaluation of drug delivery systems that release a therapeutic agent at a rhythm that ideally matches the biological requirement of a given disease therapy. "Chronopharmaceutics" consist of two words chronobiology and pharmaceutics. Chronobiology is the study of biological rhythms. There are three types of mechanical rhythms in our body.

They are:

\section{Circadia}

\section{Ultradian}

\section{Infradian.}

Circadian This word comes from Latin word "circa" means about and "dies" means day Ultrdian Oscillation of shorter duration is called as ultradian (more than one cycle per $24 \mathrm{~h}$ ) Infradian Oscillations that are longer than $24 \mathrm{~h}$ (less than one cycle per day). Diseases where a constant levels of drug the are not preferred, but needs a pulse of therapeutic concentration in a periodic manner acts as a push for development of "Pulsatile Drug Delivery Systems"

\section{MERITS ${ }^{5}$ :}

Predictable, reproducible and short gastric residence time

Less inter- and intra-subject variability

Improve bioavailability

Limited risk of local irritation

No risk of dose dumping

Flexibility in design

Improve stability 


\section{DEMERITS ${ }^{5}$ :}

Lack of manufacturing reproducibility and efficacy

Large number of process variables

Batch manufacturing process

Higher cost of production

Trained/skilled personal needed for manufacturing

\section{NECESSITIES OF PULSATILE DDS ${ }^{6}$ :}

\section{First pass metabolism:}

Some drugs, such as beta blockers, and salicylamide, undergo extensive first pass metabolism and require fast drug input to saturatemetabolizing enzymes in order to minimize pre-systemic metabolism. Thus, a constant/sustained oral method of delivery would result in reduced oral bioavailability.

\section{Biological tolerance:}

Drug plasma profiles are explain by a decline in the pharmacotherapeutic effect of the drug, e.g., biological tolerance of transdermal nitroglycerin, salbutamol sulphate.

\section{Special chronopharmacological needs:}

Circadian rhythms in certain physiological functions are well established. It has been recognized that many symptoms and onset of disease occur during specific time periods of the 24 hour day, e.g., asthma and angina pectoris attacks are most frequently in the morning hours.

\section{Local therapeutic need:}

For the treatment of local disorders such as inflammatory disease, the delivery of compounds to the site of inflammation with no loss due to absorption in the small intestine is highly desirable to achieve the therapeutic effect and to minimize side effects.

5. Gastric irritation or drug instability in gastric fluid: Protection for those drugs are essential which are effected by gastric acidic environment and undergo degradation in gastric acidic medium (eg, peptide drugs), irritate the gastric mucosa (NSAIDS) or induce nausea and vomiting.

\section{Classification of pulsatile drug delivery systems ${ }^{6,7}$}

Pulsatile drug delivery systems (PDDS) is classified in two systems one is site-specific and other is timecontrolled systems. Drug release from site-specific systems depends on the environment in the gastro intestinal track, e.g., on $\mathrm{pH}$, presence of enzymes etc. In contrast, time-controlled DDS are independent of the biological environment. The drug release is controlled only by the system. Time-controlled pulsatile delivery has been achieved mainly with drug containing cores, which are covered with release-controlling layers Figure 1.

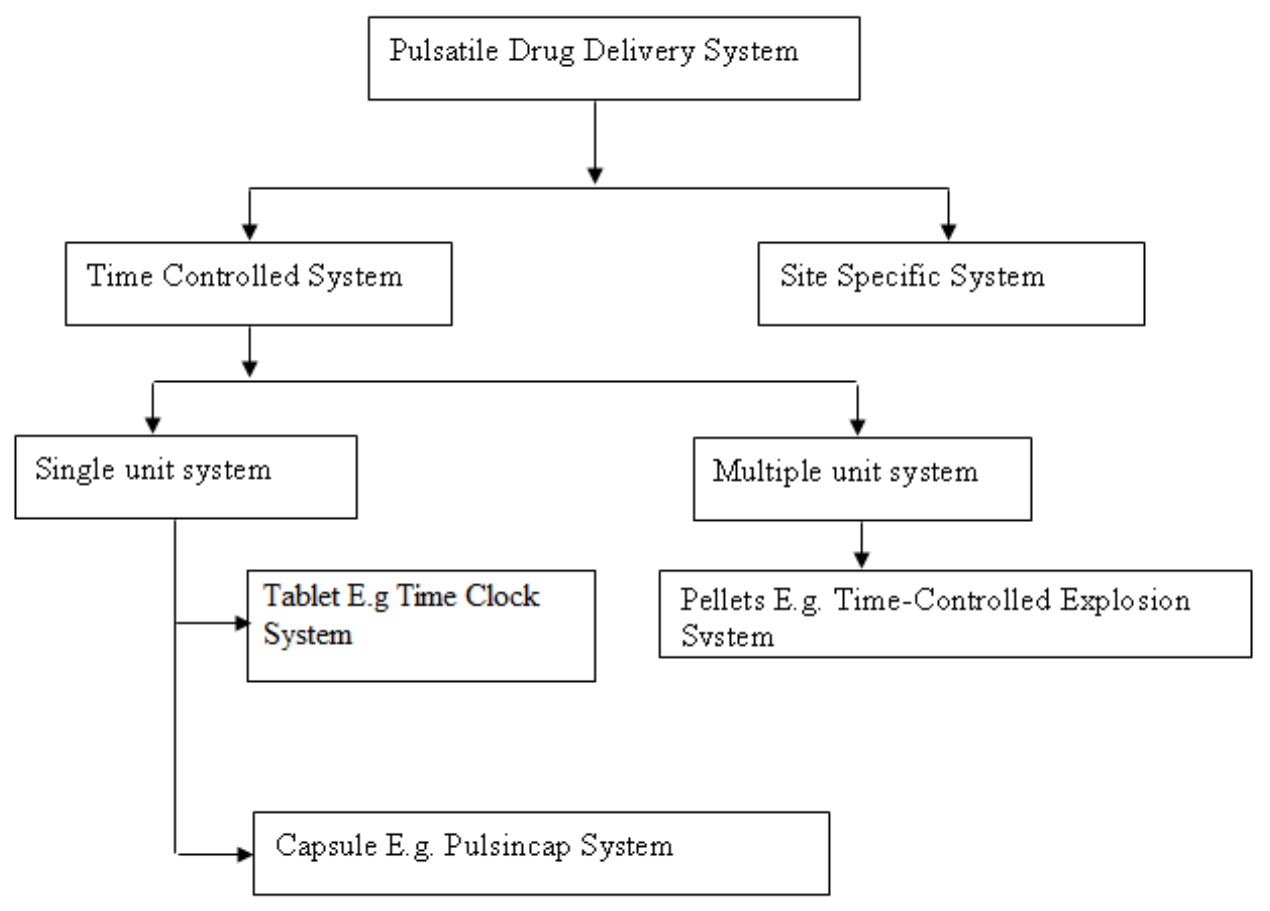

Figure 1: Classification of Pulsatile drug delivery system ${ }^{8}$

\section{TIME-CONTROLLED SYSTEM}

Time-dependent dosage forms are those dosage forms which are formulated to release their drug load after a predetermined lag time. To achieve a drug release that is independent of the environment (e.g. pH, enzymatic activity, intestinal motility) and/or other stimuli, the lag time prior to the release of the drug has to be controlled primarily by the delivery system ${ }^{8}$. The release mechanisms employed include bulk erosion of polymers in which drug release by diffusion is restricted, surface erosion of layered devices composed of alternating drug- 
containing and drug-free layers, and osmotically controlled rupture ${ }^{9}$.

\section{SITE-SPECIFIC SYSTEM}

colon specific dosage forms have been developed including pro-drugs, drug embedded in cross-linked hydrogel delivery of drugs to the colon can be done to achieve many objectives like reduction in dosing frequency, to deliver drug to a region that is less hostile metabolically efficacy of the drug generally depends upon the ability of the drug to reach its target in sufficient quantity to maintain therapeutic levels for the desired time period. Although, the colon does not inherently possess the ideal anatomical and physiological features, it is the site of significant absorption. etc ${ }^{10}$. Other commercial benefits can be the ever-greening of already existing patents and the ability to promote new claims. In the past few years many matrices, coated dosage forms, osmotic controlled drug delivery systems and timed release systems. The coated dosage forms are preferred because of innovations in the coating technology and wide flexibility in the design ${ }^{11}$. A study on several examples for both single and multiple unit gastroretentive drug delivery systems has been done by Streubel et $\mathrm{al}^{12}$ The rationale of drug targeting to the colon which we would be focusing on in this review is to delay delivery to a time appropriate to treat acute phases of a disease i.e., to achieve a chronotherapeutic drug delivery response.

\section{TIME CONTROLE SYSTEM}

\section{A. SINGLE UNIT SYSTEM}

These are sub-classified as capsule-based systems, osmotic systems.Delivery systems with soluble or erodible membranes and delivery systems with rupturable coating.

\section{(i) TABLET SYSTEM}

Most of the pulsatile drug delivery systems are reservoir devices coated with a barrier layer. This barrier erodes or dissolves after a specific lag period, and the drug is subsequently released rapidly. The lag time depends on the thickness of the coating layer.

\section{(ii) CAPSULAR SYSTEM}

Single unit systems are mostly developed in capsule form. The lag time is continued by a plug, which gets pushed away by swelling or erosion, and the drug is released as a pulse from the insoluble capsule body. e.g.: Pulsincap ${ }^{\circledR}$ system In this system a water insoluble body containing the drug formulation, system is closed with a swellable hydrogel. Plugged (insoluble but permeable \&swellable) at open end. For rapid release of water insoluble drug effervescent or disintegrating agents are added ${ }^{13}$.

\section{(iii) PULSATILE DELIVERY BY OSMOSIS}

This system consists of a capsule coated with a semi permeable membrane. Inside the capsule the drug formulation.

\section{(iv) PULSATILE DELIVERY BY} SOLUBILISATION (was an insoluble plug consisting of osmotically active agent and the drug formulation ${ }^{14}$.
This system shows good invivo and invitro correlation in humans and used to deliver methylphenidate to school age children for the treatment of Attention Deficit Hyper activity Disorder (ADHD),

e.g.: Port ${ }^{\circledR}$ System Another system is also based on expendable orifice that contain capsular system in which liquid drug is absorbed on highly porous particles. Drug releases through orifice of a semi permeable capsule supported by an expending osmotic layer after the barrier layer is dissolved ${ }^{15}$. The Port ${ }^{\circledR}$ System (Port Systems, LLC) consists of a gelatin capsule coated with a semi permeable membrane (e.g., cellulose acetate) housing an insoluble plug (e.g., lipidic) and an osmotically active agent.

\section{EROSION OF MEMBRANE}

These systems are based up on a drug reservoir surrounded with a soluble or erodible barrier layer that dissolves with time and the drug releases at once after the lag time. E.g. Time Clock ${ }^{\circledR}$ system. The Time Clock system consists of solid dosage form coated with lipid barriers such as carnauba wax \& beeswax along with surfactants like polyoxy ethylene sorbinmono oleate. When this system comes in contact with the aqueous medium the coat emulsifies or erodes after the lag-time depending on the thickness of coat. The lag time of system independent of the gastrointestinal motility, $\mathrm{PH}$, enzyme $\&$ gastric residence ${ }^{16}$.

\section{(v) PULSATILE DELIVERY BY RUPTURE OF MEMBRANE}

These systems are based up on a reservoir system coated with a rupturable membrane. The outer membrane ruptures due to the pressure developed by effervescent agents (or) swelling agent ${ }^{17}$. Citric acid \& sodium bicarbonate is incorporated as effervescent mixture in tablet core coated with ethyl cellulose, when system comes in contact with water it produces carbon dioxide gas which exerts pressure $\&$ after lag time rupture the membrane \& rapid release of drug occurs . A reservoir system with a semi permeable coating is proposed especially with drugs with high first pass effect in order to obtain in-vivo drug pattern similar to the administration of several immediate release doses croscarmellose sodium starch glycollate or low substituted hydroxy propyl cellulose were used as swelling substances, which resulted in complete film rupture followed by rapid drug release. The lag time is controlled by composition of outer polymeric membrane.

\section{B. MULTIPAL UNIT SYSTEMS}

Multiparticulate systems are reservoir type of devices with a coating, which either ruptures or changes its permeability. Drug is coated over sugar seeds these granules may then be packaged in a capsule or compressed with additional excipients to form a tablet. The active pharmaceutical ingredient may also be blended or granulated with polymers before coating to provide an additional level of control. However, drug loading in this type of system is low due to higher need of excipients. 


\section{(i) PULSATILE DELIVERY BY RUPTURABLE COATING}

Similar to single unit system, the rupturing effect is achieved by coating the individual units with effervescent (or) swelling agents. Drug deliver was controlled by the rupture of the membrane. The timing of release was controlled by the thickness of coating and the amount of water soluble polymer to achieve the pulsed release. The swelling agent includes super disintegrents like carboxymethylcellulose, sodium starch glycollate, and Lhydroxy propyl cellulose. Polymers like polyacrylic acid, polyethylene glycol etc. alternatively comprising of a mixture of tartaric acid \& sodium bicarbonate that used as effervescent agent ${ }^{18}$. The lag time is controlled by composition of outer polymeric membrane.

\section{METHODOLOGIES FOR PULSATILE DRUG DELIVERY SYSTEM}

Methodologies for the pulsatile drug delivery system can be broadly classified into three classes:

1. Time controlled

2. Stimuli induced

3. Externally regulated

4. Multipaticulate system

\section{TIME CONTROLLED PULSATILE RELEASE SYSTEM}

In time controlled drug delivery systems pulsatile release is obtained after a specific time interval in order to mimic the circadian rhythm. Such type of pulsatile drug delivery system contains two Components: one is of immediate release type and other one is a pulsed release type. Various methodologies that can be used for time controlledpulsatile release systems are discussed in following section.

\section{(i) Delivery systems with rupturable coating layer}

These systems consist of an outer release controlling water insoluble but permeable coating subject to mechanically induced rupture phenomenon. Recently different systems based on hard gelatin capsules, all coated by inner swellable and outer rutpurable layer. The film rupture may be attained by including swelling, osmotic oreffervescent additives in the reservoir. By optimizing the system, drug release can be obtained at specific time interval.

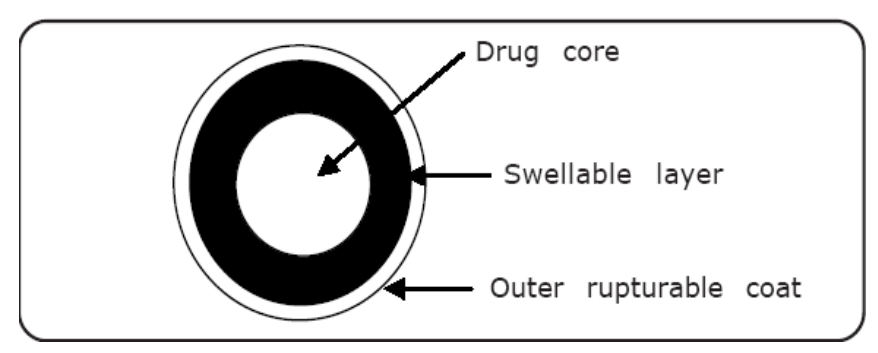

Figure 2: Schematic Diagram of delivery System with rupturable coating $\mathrm{drug}^{7}$

(ii) Delivery systems provided with erodible coating layers
In such systems the drug release is controlled by the dissolution or erosion of the outer coat which is applied on the core containing drug. Time dependent release of the active ingredient can be obtained by optimizing the thickness of the outer coat. Sangalliet al. developed an oral dosage form devised to release drugs following a programmed time period after administration based on this concept.

(iii) Capsule shaped system provided with release controlling plug

These systems contain release controlling plug between immediate release compartment and pulsed release compartment. On contact with aqueous fluids, the cap rapidly dissolves thereby releasing the immediate release component followed by pulsed release component. The lag time is provided by the plug which is inserted in to the body. In an approach used by Jimoh et al, pulsatile release was achieved by generation of hydrostatic pressure inside the capsule.

\section{STIMULI INDUCED PULSATILE SYSTEMS}

In these systems there is release of the drug after stimulation by any biological factor like temperature, or any other chemical stimuli. These systems are further classified in to temperature induced systems and chemical stimuli induced system, on the basis of stimulus.

\section{(i) Temparature induced systems}

Thermo-responsive hydrogel systems have been developed for pulsatile release. In these systems thepolymer undergoes swelling or deswelling phase in response to the temperature which modulate drug release in swollen state ${ }^{18}$. Y.H. Bae et al developed indomethacin pulsatile release pattern in the temperature ranges between $200 \mathrm{C}$ and $300 \mathrm{C}$ by using reversible swelling properties of copolymers of $\mathrm{N}$-isopropylacrylamide and butyrylacrylamide. Kataokaet al developed the thermo sensitive polymeric micelles as drug carrier to treat the cancer.

\section{(ii) Chemical stimuli induced pulsatile systems}

\section{(a) Glucose-responsive insulin release devices}

In case of diabetes mellitus there is rhythmic increase in the levels of glucose in the body requiring injection of the insulin at proper time. Several systems have been developed which are able to respond to changes in glucose concentration. One such system includes $\mathrm{pH}$ sensitive hydrogel containing glucose oxidase immobilized in the hydrogel. When glucose concentration in the blood increases glucose oxidase converts glucose into gluconic acid which changes the $\mathrm{pH}$ of the system. This $\mathrm{pH}$ change induces swelling of the polymer which results in insulin release. Insulin by virtue of its action reduces blood glucose level and consequently gluconic acid level also gets decreased and system turns to the deswelling mode thereby decreasing the insulin release. Examples of the $\mathrm{pH}$ sensitive polymers includes $N$,Ndimethylaminoethylmethacrylate. 


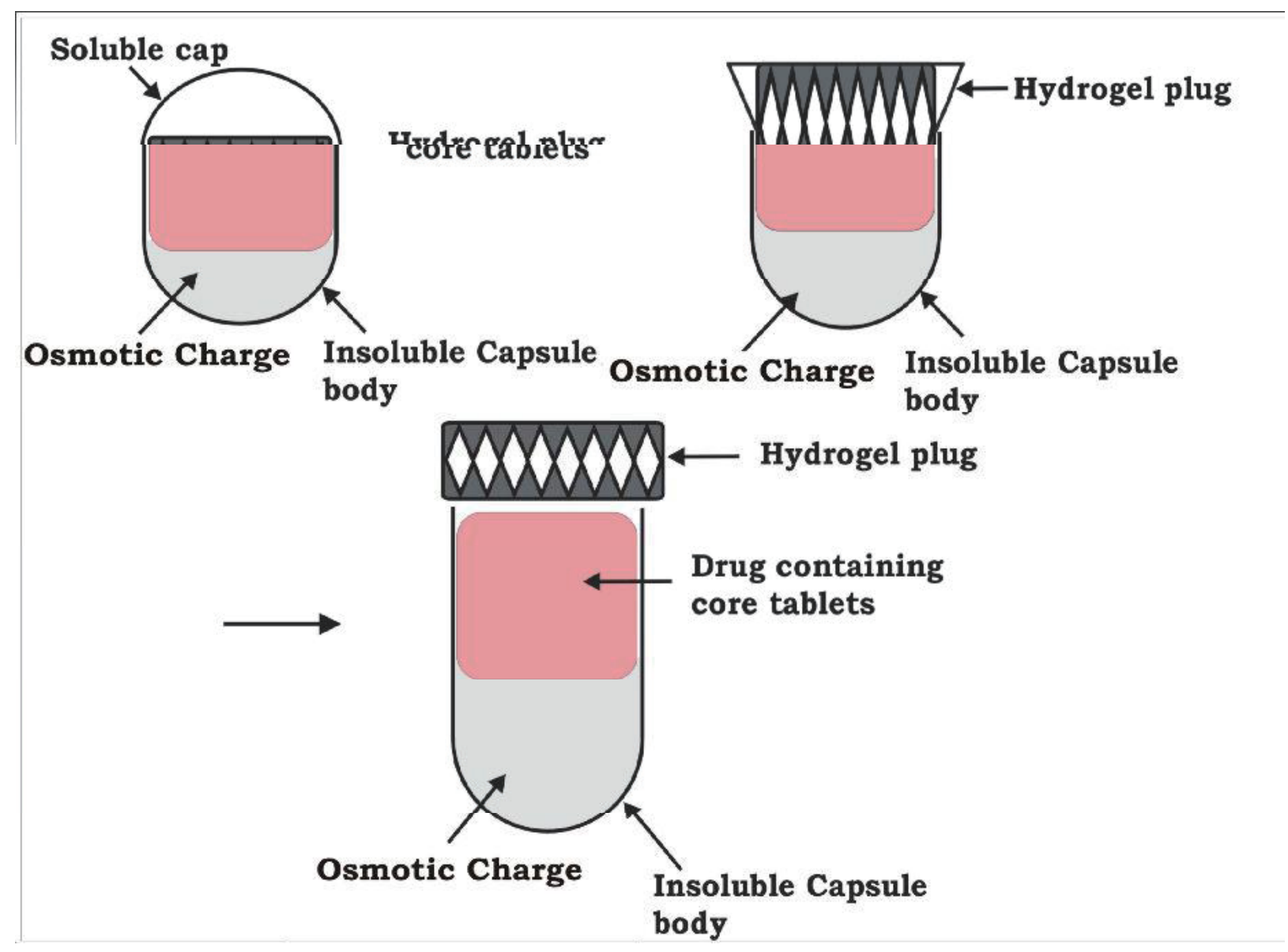

Figure 3: Schematic diagram of drug delivery with release controlling plug ${ }^{5}$

(b) Inflammation-induced pulsatile release

On receiving any physical or chemical stress, such as injury, fracture etc., inflammation take place at the injured sites.During inflammation, hydroxyl radicals are produced from these inflammation-responsive cells. Yui and co-workers focused on the inflammatory inducedhydroxyl radicals and designed drug delivery systems, which responded to the hydroxyl radicalsand degraded in a limited manner.

(c) Drug releasefrom intelligent gels responding to antibody concentration

There are numerous kinds of bioactive compounds which exist in the body. Recently, novel gels were developed which responded to the change in concentration of bioactive compounds to alter their swelling/deswelling characteristics. Special attention was given to antigenantibody complex formation as the cross-linking units in the gel, since such interaction is very specific.

\section{(d) PH sensitive drug delivery system}

Such type of pulsatile drug delivery system contains two components one is of immediate release type and other one is pulsed release which releases the drug in response to change in $\mathrm{pH}$. In case of $\mathrm{pH}$ dependent system advantage has been taken of the fact that there exists different $\mathrm{pH}$ environment at different parts of the gastrointestinal tract. By selecting the $\mathrm{pH}$ dependent polymers drug release at specific location can be obtained. An example of $\mathrm{pH}$ dependent polymers includes cellulose acetate phthalate,polyacrylates, and sodiumcarboxymethylcellulose. Externally regulated systems for releasing the drug in a pulsatile manner, another way can be the externally regulated systems in which drug release is programmed by external stimuli likemagnetism, ultrasound, electrical effect and irradiation.Magnetically regulated systems contain magnetic beads in the implant. On application of the magnetic field, drug release occurs because of magnetic beads.

\section{(iii) Magnetic stimuli-induced pulsatile release}

Use of an oscillating magnetic held to regulate the rates of drug delivery from a polymer matrix was one of the first methodologies investigated to achieve an externally controlled drug delivery system. Magnetic steel beads were engrafted in an ethylene and vinyl acetate (EVAc) copolymermatrix that was loaded with bovine serum albumin as a model drug. Edelman and coworkersproved increased rates of drug release in the presence of an oscillating magnetic field.The beads oscillate within the matrix on exposure to the magnetic field, alternatively creatingcompressive and tensile forces. This in turn acts as a pump to push more amount of the activesolute out of the matrix. Co-polymers having higher Young's modulus were more resistant tothe induced motion of steel beads, and accordingly the magnetic held has less effect onthe rate of drug release from these materials. Saslawski and co-workers prepared various formulations for in vitro magnetically induced pulsatile delivery of insulin based on alginate spheres. In an experiment, ferrite 
microparticles $(1 \mu \mathrm{m})$ and insulin powder were dispersed in sodium alginate aqueous solution.

\section{(iv) Pulsatile system based on osmosis}

The delivery of agents from osmotic systems is based on expandable orifice technology was developed. This system is in the form of capsule with an orifice.The capsule wall is constructed of an elastic material (e.g., styrene-butadiene copolymer), and stretches under a pressure differential caused by the pressure rise inside the capsule due to osmotic infusion of moisture from the body. When this elastic wall is stretched, because of pressure differential across the wall, exceeding a threshold, the orifice expands sufficiently to allow the release of the drug at a physiologically required rate. However, when the elastic wall is relaxed, the flow rate of the drug through the orifice is substantially zero.

\section{(v) Thermoresponsive hydrogel systems:}

Thermo-responsive hydrogel systems employ hydrogels which undergo reversible volume changes in response to changes in temperature. These gels shrink at a transition temperature that is referred to the lower critical solution temperature (LCST) of the linear polymer. Thermosensitive hydrosensitive hydrogels have a certain chemical attraction for water, and therefore they absorb water and swell at temperatures below the transition temperature whereas they shrink or deswell at temperatures above the transition temperature by expelling water. Thermally responsive hydrogels and membranes have been extensively exploited as platforms for the pulsatile drug delivery ${ }^{20}$.

\section{(vi) Thermoresponsive polymeric micelle systems:}

In this type, the gel system tightly stores targeted drug in the micelles and rapidly releases controlled amount of the drug by switching on-off of external stimuli such as temperature or infrared laser beam. Jianxiang Zhang, et al synthesized thermally responsive amphiphilicpoly $(\mathrm{N}$ isopropylacrylamide) (PNIPAm)-graftedpolyphosphazene (PNIPAm-g- PPP) by stepwise cosubstitution of chlorine atoms on polymer backbones with amino-terminated NIPAm oligomers and ethyl glycinate(GlyEt). Diflunisal (DIF)-loaded micelles were prepared by dialysis method. In vitro release test at various temperatures was also performed to study the effect of temperature on the drug release profiles.

\section{(vii) EnzymaticaIIy-activated Liposome:}

Drug loaded liposomes was incorporated into microcapsules of alginate hydrogels. Liposomes inside the microcapsules were coated with phospholipase A2 to achieve a pulsatile release of drug molecules. Phospholipase A2 was shown to accumulate at the water/liposome interfaces and remove an acyl group from the phospholipids in the liposome. Destabilised liposomes release their drug molecules, thus allowing drug release to be regulated by the rate determining microcapsule membrane ${ }^{21,22}$.

\section{Externally regulated pulsatile release system:}

This system is not self-operated, but instead requires externally generated environmental changes to initiate drug delivery. These can include magnetic fields, ultrasound, electric field, light, and mechanical force.

\section{(i) Magnetic induces release:}

Magnetic carriers receive their magnetic response to a magnetic field from incorporated materials such as magnetite, iron, nickel, cobalt etc. Magnetic-sensitive behavior for controlled release of drug was studied by Tingyu Liu, et al. An intelligent magnetic hydrogel (ferrogel) was fabricated by mixing poly (vinyl alcohol) (PVA) hydrogels and $\mathrm{Fe} 3 \mathrm{O} 4$ magnetic particles through freezing-thawing $\mathrm{Cycles}^{23}$. Although the external direct current magnetic field was applied to the ferrogel, the drug got accumulated around the ferrogel, but the accumulated drug spurt to the environment instantly when the magnetic fields instantly switched "off'. Furthermore, rapid slow drug release can be tunable while the magnetic field was switched from "off" to "on" mode. The drug release behavior from the ferrogel is strongly dominated by the particle size of $\mathrm{Fe} 3 \mathrm{O} 4$ under a given magnetic field $^{24}$.Tingyu Liu, et al developed the magnetic hydrogels which was successfully fabricated by chemically crosslinking of gelatin hydrogels and Fe3O4 nanoparticles (ca. 40-60 nm) through genipin (GP) as cross-linking agent. ${ }^{25}$

\section{(ii) Ultrasound induces release:}

Ultrasound is mostly used as an enhancer for the improvement of drug permeation through biological barriers, such as skin. The interactions of ultrasound with biological tissues is divided into two broad categories: thermal and nonthermal effects. Thermal effects are associated with the absorption of acoustic energy by the fluids or tissues ${ }^{26}$. Non-thermal bio-effects are generally associated with oscillating orcavitating bubbles, but also include noncavitation effects such as radiation pressure, radiation torque, and acoustic streaming.

\section{(iii) Electric field induces release:}

Electrically responsive delivery systems are prepared by polyelectrolytes (polymers which contain relatively high concentration of ionisable groups along the backbone chain) and are thus, pH-responsive as well as electroresponsive. Under the influence of electric field, electroresponsive hydrogels generally bend, depending on the shape of the gel which lies parallel to the electrodes whereas deswelling occurs when the hydrogel lies perpendicular to the electrodes.

\section{(iv) Light induces release:}

Light-sensitive hydrogels have potential applications in developing optical switches, display units, and opthalmic drug delivery devices ${ }^{27}$. The interaction between light and material can be used to modulate drug delivery. When hydrogel absorb the light and convert it to heat, raising the temperature of composite hydrogel above its LCST41, hydrogel collapses and result in an increased rate of release of soluble drug held within the matrix.

\section{Multipaticulate system:}


Recent trends indicate that multiparticulate drug delivery systems are especially suitable for achieving controlled or delayed release oral formulations with low risk of dose dumping, flexibility of blending to attain different release patterns as well as reproducible and short gastric residence time. Such systems are reservoir type with either rupturable or altered permeability coating and generally housed in capsular body. The purpose of designing multiparticulate dosage form is to develop a reliable formulation that has all the advantages of a single unit formulation and yet devoid of the danger of alteration in drug release profile and formulation behaviourdueto unit to unit to unit variation ${ }^{28}$.

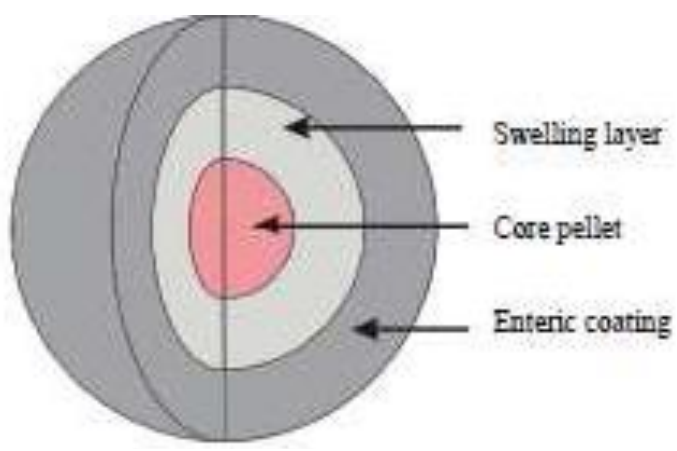

Figure 4: Hypothetical design of a multiparticulate pulsatile system $^{6}$

Andrei Dashevsky, et al. developed a pulsatile multiparticulate drug delivery system (DDS), coated with aqueous dispersion of Aquacoat ${ }^{\circledR}$ ECD. A rupturable pulsatile drug delivery system consists of (i) a drug core; (ii) a swelling layer, comprising a superdisintegrant and a binder; and (iii) an insoluble, water-permeable polymeric coating $^{29}$. Upon water ingress, the swellable layer expands, resulting in the rupturing of outer membrane with subsequent rapid drug release. Regarding the cores, the lag time was shorter; theophylline was layered on sugar cores compared with cores consisting of theophylline. Regarding swelling layer, the release after lag time was fast and complete. Drug release was achieved after the lag time, when low-substituted hydroxypropyl cellulose (L-HPC) and sodium starch glycolate(Explotab®) were used as swelling agents. Outer membrane, formed using aqueous dispersion Aquacoat ${ }^{\circledR}$ ECD was brittle and ruptured sufficiently to ensure fast drug release, compared to ethylcellulose membrane formed using organic solution. The addition of talc led to increase brittleness of membrane and was very advantageous. Drug release starts only after rupturing of outer membrane $\mathrm{e}^{23}$.

\section{EVALUATION OF PULSATILE DRUG DELIVEY SYSTEM}

\section{In vitro method}

\section{Dissolution studies}

Dissolution studies were carried out by using USB Paddle dissolution test apparatus. Capsule were tried to paddle with a cotton thread so that the capsule should be immersed completely in dissolution media but not float. In order to stimulate the PH changes along the GIT tract, three dissolution media with $\mathrm{PH} 1.2,7.4$, and 6.8 were sequentially used, reffered to as sequential $\mathrm{PH}$ change method when performing experiments, the PH 1.2 Medium was first used for $2 \mathrm{~h}$ (since the average gastric emptying time is $2 \mathrm{~h}$ ), then removed and the first PH 7.4 phosphate buffer saline (PBS) was added. After $3 \mathrm{~h}$ (average small intestinal transit time is $3 \mathrm{~h}$ ), the medium was removed and fresh PH 6.8 Dissolution medium was added for subsequent hours. Nine hundred milliliters of the dissolution medium was used at each time. Rotation speed was $100 \mathrm{rpm}$ and temperature was maintained. Capsule were tried to paddle with cotton thread in each dissolution vessel to prevent floating. Five milliliters of dissolution media was replaced. The withdrawn sample were analyzed at $296 \mathrm{~nm}$, by UV absorption spectroscopy and the cumulative percentage release was calculated over the sampling times ${ }^{26}$.

Table 1: In-vivo method

\begin{tabular}{|c|c|c|c|}
\hline S.No. & Drug (polymer) & Method & Ref \\
\hline 1. & $\begin{array}{l}\text { Verapamil hydrochloride, } \\
\text { hydroxypropyl methylcellulose } \\
\text { (HPMC, Methocel® E5, E15, E50), } \\
\text { ethylcellulose (EC, Ethocel® 45P) }\end{array}$ & $\begin{array}{l}\text { Gamma scintigraphy: six healthy males administrated two capsules } \\
\text { eachvolunteer, } 4 \mathrm{~h} \text { after dinner and then remained supine } 8 \mathrm{~h} \\
\text { following the dose, with } 200 \mathrm{ml} \text { of water containing } 99 \mathrm{mTc} \text {-DTPA. } \\
\text { The images taken at } 30 \text {-min intervalsby a gamma camera the } \\
\text { gastrointestinal transit of the dosage forms }\end{array}$ & 28 \\
\hline 2. & $\begin{array}{l}\text { Dofetilide (PulsincapTM), } \\
\text { EC, polyethylene glycol }\end{array}$ & $\begin{array}{l}\text { An open, four-way crossover study in which male fasted subjects. } \\
\text { In additionto the three doses of dofetilide administered to eleven } \\
\text { subjects gamma camerafitted with a medium energy collimator, } \\
\text { scintigraphic images of } 30 \mathrm{~s} \text { duration.Each imaging interval a blood } \\
\text { sample }(5 \mathrm{ml}) \text { was taken from a forearm vein. }\end{array}$ & 29 \\
\hline 3. & $\begin{array}{l}\text { Metoprolol tartrate, } \\
\text { impermeable capsule }\end{array}$ & $\begin{array}{l}\text { Two male and two female mongrel dogs administered hard gelatin } \\
\text { capsulespacked with two doses }(50 \mathrm{mg}+120 \mathrm{mg}) \text { were studies } \\
\text { carried out on over nightfasting condition. Plasma samples were } \\
\text { taken by repeated. }\end{array}$ & 30 \\
\hline 4. & $\begin{array}{l}\text { Acetaminophen, Methocel } \AA \text { E5, } \\
\text { Aquacoat } \AA, \text { ECD }\end{array}$ & $\begin{array}{l}\text { Five healthy volunteers administered pellets with with } 250 \mathrm{ml} \text { water } \\
\text { after a } 10 \mathrm{~h} \text { overnight fasting. Saliva collected at predetermined time } \\
\text { intervals were keptfrozen at } 60^{\circ} \mathrm{C} \text { until analytical assessment. }\end{array}$ & 31 \\
\hline
\end{tabular}




\section{APPLICATION OF PULSATILE DRUG DELIVERY SYSTEM}

Thorough understanding of the disease physiology is required before designing the pulsatile drug delivery system. Diseases where rhythmic circadian organization of the body plays an important role, pharmacokinetics and/or pharmacodynamics of the drugs is not constant within $24 \mathrm{~h}$. Table enumerates various diseases showing such a chronological behavior. Asthma is one such disease where pulsatile drug delivery system can be useful. Circadian changes are seen in normal lung function, which reaches a low point in the early morning hours. In case of cardiovascular diseases, several functions (e.g. BP, heart rate, stroke volume, cardiac output, blood flow) of the cardiovascular system are subject to circadian rhythms. For instance, capillary resistance and vascular reactivity are higher in the morning and decrease later in the day ${ }^{28,30}$.

Table 2: Diseases requiring pulsatile drug delivery

\begin{tabular}{|l|l|l|}
\hline Disease & Chronological behavior & Drug used \\
\hline Peptic Ulcer & Acid secretion is high in the afternoon and at night & $\mathrm{H}_{2}$ blockers \\
\hline Asthma & Precipitation of attacks during or at early morning hour & $\beta_{2}$ agonist, Antihistaminics \\
\hline Cardiovascular diseases & $\begin{array}{l}\text { BP is at its lowest during the steep cycle and rises } \\
\text { steeply during the early morning awakening period }\end{array}$ & $\begin{array}{l}\text { Nitroglycerin, Calcium Channel } \\
\text { blocker, ACE inhibitors etc }\end{array}$ \\
\hline Arthritis & Pain in the morning and more pain at night & NSAIDs, Glucocorticoids \\
\hline Diabetes Mellitus & Increase in the blood sugar level after meal & Sulfonylurea, Insulin, Biguanide \\
\hline Attention deficit syndrome & Increase in DOPA level in the afternoon & Methylphenidate \\
\hline Hypercholesterolemia & $\begin{array}{l}\text { Cholesterol synthesis is generally higher during night } \\
\text { than during day time }\end{array}$ & HMG CoA reductase inhibitors \\
\hline
\end{tabular}

\section{RECENT ADVANCES IN THE PULSATILE DRUG DELIVERY}

Nowadays pulsatile drug delivery systems are gaining importance in various disease conditions specifically in diabetes where dose is required at different time intervals. Among these systems, multi-particulate systems (e.g. pellets) offer various advantages over single unit which include no risk of dose dumping, flexibility of blending unitswith different release patterns, as well as short and reproducible gastric residence time. Multiparticulate systems consists pellets of different release profile which can be of any type like time dependent, $\mathrm{pH}$ dependent, micro flora activated system as discussed in the previous sections. Site and time specific oral drug delivery have recently been of great interest in pharmaceutical field to achieve improved therapeutic efficacy. Gastroretentive drug delivery system is an approach to prolong gastric residence time, thereby targeting sitespecific drug release in upper gastrointestinal (GI) tract. Floating drug delivery system (FDDS) and bioadhesive drug delivery are widely used techniques for gastro retention. Low density porous multiparticulate systems have been used by researchers for formulation of FDDS. Sharma and Pawar developed multiparticulate floating pulsatile drug delivery system

\section{REFFERENCES}

1. Kikuchi A, Okano T, Pulsatile drug release control using hydrogels, Advance Drug Delivery Reviews, 2002, 54, 53-77.

2. Rubinstein A, Tirosh B, Baluom M, Nassar T, David A, The rationale for peptide drug delivery to the colon and the potential of polymeric carriers as effective tools, J. Controlled Release, 46, 1995,59-73.

3. Pozzi F, Furlani P, Gazzaniga A, Davis SS, Wilding IR, The time clock system: a new oral dosage form for fast and complete release of drug after a predetermined lag time, J. Controlled Release, 1994, 31, 99-108. using porous calcium silicate and sodium alginate for time and site specific drug release of meloxicam. Various pulsatile technologies have been developed on the basis of methodologies as discussed previously. These includes OROS® technology, CODAS $®$ technology, CEFORM® technology, DIFFUCAPS $®$ technology, Threedimensional printing $₫$, timerx ${ }^{\circledR}$ etc $^{22,27,30}$.

\section{CONCLUSIONS}

There is a need of new drug delivery systems that can provide increased therapeutic benefits to the patients. Pulsatile drug delivery system (PDDS) is one such system that delivers the drug at the right time, right place and in right amounts, holds good promises of benefit to the patients suffering from chronic problems . Thus, designing of proper pulsatile drug delivery will enhances the patient compliance, optimum drug delivery to the target site and minimizes the undesired effects. PDDS can effectively tackle the problems as it is modulated according to body's circadian clock giving release of drug after a specified time lag. Significant progress has been made towards achieving pulsatile drug delivery system that can effectively treat diseases with non-constant dosing therapy.

4. Ronald AS, Colin GP, A strategy for oscillatory drug release general scheme and simplified theory, J. Controlled Release, 1995, 33, 173-188.

5. Dalvadi H, Patel JK, Chronopharmaceutics, Pulsatile Drug Delivery System as current trend, Asian Journal of Pharmaceutical Sciences, 2010,5(5),204-230.

6.Rasve G, Borade G, Siddheshwar D, Tagalpallewar A, Pulsatile Drug Delivery System:Current scenario, IJPBS, 2011, 2(3), 332-343.

7. Gothoskar AV, Joshi AM, Joshi NH, Pulsatile Drug Delivery Systems: A Review, Drug Delivery Technology, 2004, 4(5), 3142.

ISSN: 2250-1177 
8. Shivakumar HG, Pramod kumar TM , Desai KG, Pulsatile drug delivery system, Indian J Pham Educ. 2003,37(3), 125.

9. Cohen S, Bernstein H (Eds). Microparticulate systems for the delivery of proteins and vaccines. New York, Marcel Dekker, Inc. 1995. P. 201-216.

10. Bussemer T, Otto I, Bodmeier R, Pulsatile drug-delivery systems, Crit Rev Ther Drug Carrier Syst 2001, 18, 433-58.

11. Wilson C. Colonic drug delivery; In: Modified release drug delivery technology. New York, Marcel Dekker ;2003.P. 217223.

12. Verma R, Garg S, Current status of drug delivery technologies and future directions, Pharm Tech, 2001, 25 (2), 114 .

13.Streubel A, Siepmann J, Bodmeier R, Drug delivery to the upper small intestine window using gastroretentive technologies, Curr Opinion Pharmacol, 2006, 6, 501-508.

14. Baker RW, Controlled release delivery system by an osmotic bursting mechanism. US Patent 3,952,741, 1976.

15. Schultz PA, Kleinebudde P, New multiparticulate delayed release system. Part I: dissolution properties and release mechanism, J. Control. Release, 1997,47, 181-189.

16. Morita R, Honda Y, Takahashi R, Development of oral controlled release preparations, a PVA swelling controlled release system (SCRS). I. Design of SCRS and its release controlling factor, J. Control. Release, 2000, 63, 279-304.

17. Das NG, Das SK, Controlled release of oral dosage forms, formulation, finish, and fill, 2003, 10-16.

18. Ueda T, Hata 21. Okano T., Yuim N., Yokoyama, M. and Yoshida, R., "Pulsatile drug delivery system, year 2007,vol 69,issue 4,page 489-497.

19. Miyata T, Asami N, Uragami T, A reversibly antigenresponsive hydrogel, Nature, 1999, 399, 766-769.

20. Miyata T, Asami N, Uragami T, Preparation of an antigensensitive hydrogel using antigen-antibody bindings, Macromolecules, 1999, 32, 2082- 2084.
21. Liu TY, Hu SH, Liu TY, Magnetic-sensitive behavior of intelligent ferrogels for controlled release of drug., Langmuir, 2006, 22, 5974-5978.

22. Cai K, Luo Z, Hu Y, Magnetically triggered reversible controlled drug delivery from microfabricated polymeric multireservoir devices, Adv. Mater., 2009, 21, 4045-4049.

23.Liu TY, Hu SH, Liu KH, Preparation and characterization of smart magnetic hydrogels and its use for drug release, J. Magnetism Magnetic Materi., 2006, 304, e397-e399.

24. Wesley N, Biological effects of ultrasound: Development of safety guidelines. Part II: General review. Ultrasound Med Biol, 2001,27(3), 301-333.

25. Qiu Y, Park K, Environment-sensitive hydrogels for drug delivery, Adv. Drug Del. Reviews, 2001, 53, 321-339.

26. Averitt RD, Westcott SL, Halas NJ, Linear optical properties of gold nanoshells, J. opt Soc. Amer. B., 1996,16(10), 1824-1832.

27. Roy P, Shahiwala A, Multiparticulate formulation approach to pulsatile drug delivery: current perspectives, J. Control. Release, 2009, 134,74-80.

28. Zou H, Jiang XT, Kong LS, Design and gammascintigraphic evaluation of a floating and pulsatile drug delivery system based on an impermeable cylinder, Chem. Pharm. Bull., 2007, 55, 580-585

29. Stevens NE, Wilson CG, Welling PG, Evaluation of Pulsincap ${ }^{\mathrm{TM}}$ to provide regional delivery of dofetilide to the human GI tract. Int. J. Pharm., 2002, 236, 27-34.

30.Löbenberga R, Kim JS, Amidon GL. Pharmacokinetics of an immediate release, a controlled release and a two pulse dosage form in dogs. Eur. J. Pharm. Biopharm., 2005, 60, 17-23.

31. Sawada T, Sako K, Fukui M, A new index, the core erosion ratio, of compression-coated timed-release tablets predicts the bioavailability of acetaminophen, Int. J. Pharm., 2003, 265, 5563. 